\title{
Energy conditions in non-Riemannian spacetimes
}

\author{
Larry L. Smalley ${ }^{a}$, Jean P. Krisch ${ }^{b}$ \\ a Department of Physics, University of Alabama in Huntsville, Huntsville, AL 35899, USA \\ ' Department of Physics, University of Michigan, Ann Arbor, MI 48109, USA
}

Received 12 July 1994; revised manuscript received 20 July 1994; accepted for publication 4 November 1994

Communicated by P.R. Holland

\begin{abstract}
We interpret the energy conditions in non-Riemannian spacetimes by applying them to a family of stationary, axially symmetric solutions for the gravitational field equations for an Einstein-Cartan theory in a Riemann-Cartan spacetime.
\end{abstract}

\section{Introduction}

Energy conditions are frequently used to motivate the physical reasonableness of interior solutions to the gravitational field equations. In general relativity (GR), there are three conditions in common use $[1,2]$. One is the weak energy condition,

$$
T_{i j} u^{i} u^{j} \geqslant 0
$$

where $T_{i j}$ is the stress energy tensor and $u^{i}$ is a timelike vector. This condition is normally interpreted as requiring the energy density as measured by an observer moving with velocity $u^{i}$ to be positive. The condition may also be used to discuss the non-divergence of null geodesic congruences. A second energy condition is the dominant energy condition. Its statement requires the weak energy condition plus the additional statement that

$$
T_{i j} u^{i} T^{j k} u_{k} \leqslant 0
$$

i.e., that the local energy vector, $T_{i j} u^{i}$, be non-spacelike so that the velocity of matter flow will not exceed the speed of light $c$. The third energy condition, the strong energy condition, is

$$
\left(T_{i j}-\frac{1}{2} g_{i j} T\right) u^{i} u^{j} \geqslant 0 .
$$

This condition is sometimes used as a physical statement on the relative size of stresses versus energy content. It can also be used to discuss the focusing of timelike geodesic congruences. The interpretation of these conditions is the one usually presented for Riemannian geometry. Stress energy tensors that do not satisfy some of these conditions are usually regarded as being unphysical. As sometimes happens, the results of literally applying the energy conditions, in the form of the mathematical statements given by Eqs. (1)-(3), are used in place of the actual meaning of the conditions themselves. There has been a belief that negative energy densities always 
imply a violation of one or more of the energy conditions. Recently Parker and Wong $|3|$ and Kandrup $|4|$ have discussed violations of the strong energy conditions for GR. The question of the validity of the energy conditions in non-Riemannian geometry is of interest.

In this paper we would like to examine the energy conditions directly for an Einstein-Cartan theory in $\mathrm{RC}$ spacetime. Unfortunately the energy conditions for GR in Riemannian spacetime given by Eqs. (1)-(3) are not valid in their form for $T_{i j}$ developed for an EC theory in RC spacetime, as has been discussed, in a similar context, by Baekler and Mielke for the Poincaré gauge theory since the Hamiltonian structure in RC spacetime is different from that in Riemannian spacetime [5]. In Section 2. we will briefly discuss the results of an EC Lagrangian-based theory in RC spacetime [6]. Thus one can in principle develop a Hamiltonian for the theory which will yield the energy conditions in RC. Alternately we can always write the field equations in their "effective" Riemannian form for which Eqs. (1)-(3) would then be valid $|7.9|$ (also referred to as "pseudo-Einsteinian" in Ref. [81). We discuss this development in Section 3 and defer the Hamiltonian treatment to another work.

To facilitate our discussion, we have generated a family of stationary, axially symmetric constant curvature $\mathrm{RC}$ solutions. The solution that we will use in our example has a constant curvature general relativistic limit. There are many solutions to the field equations with torsion in the literature. Some of the earliest work has heen done by Kuchowicz [10] and Tafel [11]. More recently, Krisch and Smalley |12], using a spin fluid energy content, have extended some general relativistic solutions to spacetimes with torsion. The metric solution that we have found, since it describes a constant curvature spacetime, is not new. Such spaces are well discussed in the literature [13]. However, the matter content of our solution is new and it is that aspect of our solution which is necessary to discuss the RC energy conditions. In the next section of the paper we derive the solution and apply to the effective-Riemannian form in Section 3.

\section{Rotating cosmology in Riemann-Cartan spacetime}

This family of rotating cosmologies is based upon a Lagrangian-based, self-consistent spinning fluid description [6] for the Einstein-Cartan theory which is a generalization to RC spacetime of a Weyssenhoff fluid with spin density and particle number conservation $[14.15]$. The metric field equation takes the usual form

$$
G_{i j}-\nabla_{k}\left(S_{l i}{ }^{k}-S_{j}{ }^{k}+S^{k}{ }_{i j}\right)=k T_{i j} .
$$

where the holonomic coordinates are represented by Latin indices, $i=0, \ldots, \nabla_{k}$ is the covariant derivative in $\mathrm{RC}$ spacetime, and $k=8 \pi G$ where $G$ is the gravitational constant. The Einstein tensor is given by

$$
G_{i j}=R_{i j}-\frac{1}{2} g_{i j} R,
$$

where $R_{i j}$ is the Ricei tensor, $g_{i j}$ is the metric tensor with signature $\left(-1,1,1,1\right.$, and $R=g_{i j} R^{i j}$ is the Riemann scalar. The torsion tensor is given by

$$
S_{i j}^{k}=\gamma_{|i j|}^{k} \text {. }
$$

where the connection

$$
\Gamma_{i j}{ }^{k}=\left\{i^{k}\right\}+S_{i j}{ }^{k}-S_{j}{ }^{k} i+S^{k}{ }_{i j}
$$

and $\left\{\begin{array}{c}k \\ i, j\end{array}\right\}$ is the Christoffel connection. The self-consistent energy momentum tensor for a spinning fluid is

$$
T_{i j}=T_{i j}^{\mathrm{F}}+T_{i j}^{\S},
$$

with perfect fluid part

$$
T_{i j}^{1}=\left\lfloor\rho(1+\varepsilon)+p \mid u_{i} u_{j}+p g_{i j}\right.
$$


and spin density part

$$
T_{i, j}^{\mathrm{S}}=2 \rho u_{(i} s_{j) k} \dot{u}^{k}+\nabla_{k}\left(\rho u_{(i} s_{j)}{ }^{k}\right)-\rho \omega_{k(i} s_{j)}{ }^{k},
$$

where $u^{i}$ is the four-velocity, $p$ is the matter pressure, $\rho$ is the matter energy density, $\varepsilon$ is the internal energy, and the four-acceleration, $\dot{u}^{k}=u^{n} \nabla_{n} u^{k}$, is the covariant directional derivative along world lines. The spin density is given by

$$
S_{i j}=\rho s_{i j},
$$

where [14]

$$
s_{i j}=k(x)\left(a_{i}^{1} a^{2}{ }_{j}-a^{1}{ }_{j} a_{i}^{2}\right),
$$

and $k(x)$ is a covariantly constant scalar function, and $a^{\mu}{ }_{i}$ is a set of tetrads that satisfy

$$
a^{\mu}{ }_{i} a_{\mu j}=g_{i j}
$$

and

$$
a_{\mu k} a_{\beta}{ }^{k}=\eta_{\mu \beta},
$$

where $a^{4 i}=u^{i}$, and $\eta_{\mu \beta}, \mu=1, \ldots, 4$ are the anholonomic (Minkowski) metric and coordinates, respectively. The tetrad angular velocity is given by

$$
\omega_{i j}=\dot{a}^{\mu}{ }_{i} a_{\mu j} \text {. }
$$

The torsion field equation is given by $[6,15]$

$$
S_{i j}{ }^{k}=\frac{1}{2} \kappa S_{i j} u^{k} .
$$

Note that the relative simplicity of the field equations, Eqs. (4) and (16), is due to the particular constraint imposed by particle number conservation which forces the torsion vector to vanish.

The form of our stationary metric in spherical coordinates is given by

$$
\mathrm{d} s^{2}=-f \mathrm{~d} t^{2}-2 k \mathrm{~d} t \mathrm{~d} \phi+\ell \mathrm{d} \phi^{2}+\mathrm{e}^{\mu} \mathrm{d} r^{2}+\mathrm{e}^{b} \mathrm{~d} \theta^{2}
$$

in terms of the unknown functions $f, k, \ell, \mu$, and $b$. In the axially symmetric solution given here, we have further imposed the constraint that both the vorticity vector, $\Omega_{k}$, and the spin density vector, $S_{k}$, be polarized along the axis of rotation given by $\theta=0$, where

$$
\Omega_{k}=g_{k \ell}(-g)^{-1 / 2} \varepsilon^{\ell m i j} u_{m} \Omega_{i j},
$$

$\varepsilon^{\ell m i j}$ is the totally antisymmetric symbol in four dimensions, and the vorticity tensor is given by (see, e.g., Ref. [16])

$$
\Omega_{i j}=-\nabla_{[j} u_{i]}-\dot{u}_{[i} u_{j]} .
$$

The vector $S_{k}$ is defined similarly to Eq. (18) in terms of the spin density given by Eq. (11).

Consistent with the above constraints, we have used the tetrads

$$
a^{\nu i}=\left[\begin{array}{cccc}
0 & \mathrm{e}^{-\mu / 2} / \sqrt{2} & \mathrm{e}^{-\beta / 2} / \sqrt{2} & 0 \\
-k / D \sqrt{f} & 0 & 0 & \sqrt{f} / D \\
0 & -\mathrm{e}^{-\mu / 2} / \sqrt{2} & \mathrm{e}^{-\beta / 2} / \sqrt{2} & 0 \\
1 / \sqrt{f} & 0 & 0 & 0
\end{array}\right],
$$


where $D^{2}=f t+k^{2}$, columns are given by the holonomic coordinate $l=(0, r, t, \phi)$. and rows are given by the anholonomic coordinate $\nu=(1,2,3,4)$. In terms of the tetrads given in Eq. (20), the non-zero components of the spin density are $S_{r \phi}$ and $S_{\theta \phi}$. Note that for polarization of the spin along the - -axis, a non-zero component Srocan not occur.

We make the following choices for the metric potentials.

$$
\begin{array}{lll}
f=r^{(k} F(\theta) . & k=r^{\gamma} G(\theta) . & \mathrm{e}^{\mu}=r^{\prime \prime} \\
\mathrm{c}^{h}=r^{h} \mathrm{e}^{\beta(t)} . & D=r^{s} D(\theta) . & t=\left(D^{2}, k^{2}\right), t .
\end{array}
$$

where $\alpha, \beta, s, m$, and $\gamma$ are constants.

We look for solutions to the RC field equations in which the spin and the vorticity are polarized along the axis of rotation and which satisfy the relation

$$
S_{r, t}=\frac{f k_{r} \cdots k f r}{f^{32}}, \quad S_{\theta \phi}=\frac{f k_{\theta}-k f_{i}}{f^{3}}
$$

Note that for polarization of the spin along the $\theta=0$ axis $(z$-axis $)$, the spin component $S_{r}$ is not allowed.

We find a variety of solutions to accommodate this spin-vorticity condition. The solution that we describe here is one which has a positive pressure but a negative energy density, since it is this kind of solution which we want to use to discuss the energy conditions. The other solutions will be reported elscwhere.

The solution we wish to examine has the parameters

$$
m=-2, \quad b=0 . \quad D(\theta)=F^{l l}(\theta) . \quad H=\frac{a \cdot s}{a} \cdot 2 s . \quad H \neq
$$

For these parameters, the equations that need to be satisfied are

$$
\frac{F^{\prime}}{F}-\frac{F^{\prime} \beta^{\prime}}{2 F}=\left(\frac{F^{\prime}}{F}\right)^{2}=\frac{\alpha \mathrm{c}^{\beta}}{(2 H-1)^{2}}
$$

and

$$
\frac{F^{\prime}}{F^{\prime}}\left(\frac{F^{\prime}}{F^{\prime}}-\frac{G^{\prime}}{G}\right) \mathfrak{e}^{\beta}=\alpha(\gamma-\alpha)
$$

For this solution the pressure and energy density are given by

$$
\cdots \rho=p=\frac{1}{2} \alpha^{2}+s^{2} \quad s \alpha .
$$

A particular solution to these equations is $H=0, \alpha=s, y=\cdots \rho=\frac{1}{2} \alpha \alpha^{2}$.

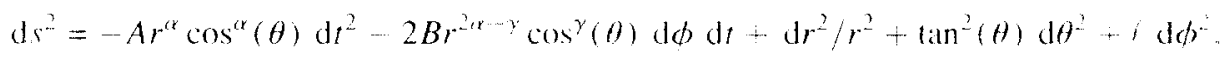

with

$$
\zeta=\left(C^{2} / A\right) r^{\alpha x} \cos ^{-\alpha}(\theta)-\left(B^{2} / A\right) r^{3 \alpha-2 \gamma} \cos ^{2 \gamma+\alpha \gamma}(\theta) .
$$

in terms of the arbitrary parameters $A, B . C$. and $\gamma$. The case $H=\frac{1}{2}$ yields a similar solution.

The solution of the field equations given by Eqs. (27), (28) self-consistently gives the magnitude of the spin density

$$
\kappa S=-\cdots 2^{1 / 2} \eta(B / C)(\alpha-\gamma) r^{\alpha-\gamma} \cos ^{\gamma}(\theta)
$$


where $\eta=\varepsilon^{0123}$, with pressure

$$
\kappa p=\frac{1}{2} \alpha^{2},
$$

and the energy density

$$
\kappa \hat{\rho}=-\frac{1}{2} \alpha^{2}
$$

where $\hat{\rho}=\rho(1+\varepsilon)$. This solution requires that the magnitude of the vorticity, given by Eq. (19), in RC spacetime vanishes, but that the magnitude of the Riemannian vorticity (using the Christoffel connection) takes the value

$$
\Omega^{\{\}}=-\frac{1}{2} \kappa S .
$$

In our solution, the overall angular momentum vanishes, but both the orbital angular momentum density and the spin density are finite. This should be contrasted with a purely rotating cosmology such as the Gödel universe [17] or a Gödel universe with non-zero Reimann-Cartan vorticity [18]. The metric equation (27) has some interesting properties. It is a space of constant curvature for all $\alpha$ and $\gamma$,

$$
R=-2 \alpha^{2}
$$

The space time is an Einstein space with

$$
R_{i j}=-\frac{1}{2} \alpha^{2} g_{i j}
$$

Because it is an Einstein space of constant curvature, it is also recurrent symmetric and Ricci recurrent [19].

The metric can be cast into an exponential form with the coordinate transformation

$$
r=a \exp \left(\frac{x+y}{\sqrt{2}}\right), \quad \cos (\theta)=b \exp \left(\frac{y-x}{\sqrt{2}}\right),
$$

with $a, b$ constant. With these tranformations the metric becomes

$$
\mathrm{d} s^{2}=-A_{1} \exp (\alpha x \sqrt{2})\left\{\mathrm{d} t+B_{1} \exp [\sqrt{2} y(\alpha-\gamma)] \mathrm{d} \phi\right\}^{2}+(\mathrm{d} x)^{2}+C_{1} \exp (\alpha y \sqrt{2})(\mathrm{d} \phi)^{2}+(\mathrm{d} y)^{2},
$$

with $A_{1}, B_{1}, C_{1}$ constants. In this form, it is clear that the GR limit of this metric $(\alpha=\gamma)$ in addition to being a constant curvature, static Einstein space, is also decomposable.

\section{Energy conditions as effective Riemannian conditions}

We have already indicated earlier that the results of applying the energy conditions is not the same as the conditions themselves. The correct interpretation of the above energy conditions in an RC spacetime as opposed to their meaning in Riemannian spacetime is not clear. We propose that in an RC spacetime, an effective stress energy tensor should be used in interpreting the energy conditions.

To this end we reexpress the RC field equations into an effective GR form with a corresponding effective energy momentum tensor. The left-hand side of the field (Eq. (4)) can be rewritten as

$$
G_{i j}-\nabla_{k}\left(S_{i j}{ }^{k}-S_{j}{ }_{i}+S^{k}{ }_{i j}\right)=G^{\left\{{ }_{i j}\right.}+\text { spin terms, }
$$

where the curly bracket in the superscript of $G$ represents the Riemannian Einstein tensor. Using this decomposition, we can write Eq. (4) in the effective Riemannian form

$$
\kappa T^{\{\}}{ }_{j k}=G^{\{\}}{ }_{j k} \equiv \kappa T^{\mathrm{RC}}{ }_{j k}-\left(2 S_{(j|x|}{ }^{m} S_{k) m}{ }^{x}+2 S_{j x}{ }^{m} S_{k}{ }_{m}^{x}-S_{m}{ }_{j} S^{m}{ }_{x k}\right)+\frac{1}{2} g_{j k}\left(S^{x}{ }_{\ell m} S_{x}^{\ell m}+2 S_{\ell x}{ }^{m} S^{x}{ }_{m}^{\ell}\right),
$$


where now $T^{\mathrm{RC}}{ }_{j k}$ is the Riemann-Cartan energy momentum tensor given by Eqs. (8)-(10) and the curly bracket in the superscript of the energy momentum tensor represents the effective Riemannian energy momentum tensor. The effective weak energy condition now becomes

$$
\kappa T^{\{\}} u_{k}^{\prime} u^{k}=-\frac{1}{2} \alpha^{2}+\frac{3}{4}(\kappa S)^{2} .
$$

which is satisfied ( i.e. $\geqslant 0$ ) for $\kappa S \geqslant\left(\frac{2}{3}\right)^{1 / 2} \alpha$. Note that this is a model dependent condition. i.e. the metric must satisfy certain boundary conditions.

Since the weak energy condition can be satisfied, it is only necessary to determine whether the vector $V_{k}=\kappa T^{\{\}}{ }_{j k} u^{j}$ can be chosen to be timelike so that the effective dominant energy condition is satisfied. After a short calculation, we find that

$$
\begin{aligned}
V^{2}= & \frac{1}{8}(\alpha-2 \gamma)^{2}(\kappa S)^{2}+\frac{1}{4}(\alpha-2 \gamma)(\alpha-\gamma)^{-1}(\kappa S)^{4}+\frac{1}{8}(\kappa S)^{\prime \prime}(\alpha-\gamma) \\
& -\left|\frac{9}{16}(\kappa S)^{4}-\frac{3}{4} \alpha^{2}(\kappa S)^{4}+\frac{1}{4} \alpha^{4}\right| .
\end{aligned}
$$

Now the weak energy condition is satistied for $\kappa S \geqslant\left(\frac{2}{3}\right)^{1-\alpha} \alpha$, we can then attempt to find a model (i.e.. adjust boundary conditions) for which $V^{2} \leqslant 0$. Such an example occurs for $\gamma=0.7 \alpha$ and $\kappa S=\left(\frac{2}{3}\right)^{1 / 2} \alpha$ for which

$$
V^{2}=-0.01 \alpha^{4}
$$

This implies that the dominant energy condition can be satisfied as well.

The effective strong energy condition is easier to satisfy. We find

$$
\kappa\left(T^{\{\}}{ }_{j k}-\frac{1}{2} \kappa T^{\{\}}\right) u^{\prime} u^{k}=-\frac{1}{2} \alpha^{2}+(\kappa S)^{2} .
$$

which is positive providing that $\kappa S \geqslant \alpha / \sqrt{2}$.

\section{Conclusions}

Although the negative energy density given by Eq. (31) is surprising, there may yet be cosmological consequences for this solution since the effective strong energy conditions can be satisfied for certain choices of the parameters in the family of solutions given by Eqs. (27), (28). It is interesting to speculate that the negative energy density in the solution presented here might suggest applications to wormholes [20].

As another approach for spinning fluids which is not simply related to the study of energy conditions applied to non-Riemannian spacetimes is to study instead a complementary problem which considers these energy conditions for self-consistent spinning fluids in GR [6]. This would involve, of course, the task of solving the field equations for that theory. However, Martins et al. [22] indicate that constraint conditions $[23,15]$ on the field equations seem to prevent meaningful cosmological solutions in GR. The solution shown in this work thereby re-emphasizes the point that the correct arena for self-consistent spinning fluids resides in RC spacetime even though one should consider effective energy conditions in order to check for viability of solutions.

\section{References}

11) S.W. Hawking and C.F.R. Ellis, The large scale structure of space-time (Cambridge Univ. Press. Cambridge 1973 ) p. $8 \times$

(2) R. Wald, General relativity (University of Chicago Press, Chicago, 1984) p. 219

131 L. Parker and Y. Wang, Phys. Rev. D 42 (1990) 1877

I 4 | H.E. Kandrup, Phys. Rev. D 46 (1992) 5360

151 P. Baekler and E.W. Mielke, Fortschr. Phys. 36 (1988) 56.5, and references therein.

[6] J.R. Ray and L.L. Smalley. Phys. Rev. Lett. 49 (1982) 1059; 50 (1982) E626: Phys. Rev. D 27 i 1983 , 1383. 
[7] F.W. Hehl, Gen. Rel. Grav. 5 (1974) 491.

[8] F.W. Hehl, P. von der Heyde and G.D. Kerlick, 10 (1974) 1066.

[9] E. Mielke, Proc. M. Grossman Meeting, 1975, ed. R. Ruffini (North-Holland, Amsterdam) p. 213

[10] B. Kuchowicz, Acta Phys. Pol. B 6 (1975) 555; 8 (1976) 81; Astrophys. Space Sci. 39 (1976) 157; 40 (1976) 167.

[11] J. Tafel, Acta Phys. Pol. B 6 (1975) 537; Phys. Lett. A 45 (1973) 341.

[12] J. Krisch and L.S. Smalley, Class. Quantum Grav. 7 (1990) 481.

[13] D. Kramer, H. Stephani, E. Herlt and M. MacCallum, Exact solutions of Einstein's field equations (1980) p. 101.

[14] F. Halbwachs, Theorie relativiste des fluides à spin (Gauthier Villars, Paris, 1960).

[15] L.L. Smalley and J.R. Ray, Gen. Rel. Grav. 18 (1986) 549.

[16] J. Ehlers and W. Kundt, in: Gravitation: An introduction to current research, ed. L. Witten (Wiley, New York, 1962 ) pp. $49-101$.

[17] O. Heckmann and E. Schücking, in: Gravitation: An introduction to current research, ed. L. Witten (Wiley, New York, 1962) pp. 442-443.

[18] L.L. Smalley, Phys. Rev. D 32 (1985) 3124.

[19] A.Z. Petrov, Einstein spaces (Pergamon, Oxford, 1969) pp. 84, 352.

[20] M.S. Morris and K.S. Thorne, Am. J. Phys. 56 (1988) 395.

[21] J.R. Ray, L.L. Smalley and J.P. Krisch, Phys. Rev. D 35 (1987) 3261.

[22] M.A.P. Martins, E.P. Vasconcellos-Vaidya and M.M. Som, Class. Quantum Grav. 8 (1991) 2225.

[23] C.W. Misner, K.S. Thorne and J.A. Wheeler, Gravitation (Freeman, San Francisco, 1973) Ch. 4. 